\title{
Implementación del sistema de inventario permanente en la ONG World Visión
}

\section{Permanent inventorysystem implementation in World Vision}

Luz Ángela Bautista Vargas * Juan David Ovalle Triana **

Como citar este artículo

Bautista, L; y Ovalle, J (2019). Implementación del sistema de inventario permanente en la ONG World Visión. Revista Colombiana de Ciencias Administrativas 1(1), pp. 46 55. Recuperado a partir de http://cipres.sanmateo.edu.co/index.php/rcca

\footnotetext{
* Estudiante, Fundación Universitaria San Mateo, Facultad Ciencias Administrativas y afines, Contaduría Pública, Correos Electrónico: langelabautista@sanmateo.edu.co

${ }^{* *}$ Estudiante, Fundación Universitaria San Mateo, Facultad Ciencias Administrativas y afines, Contaduría Pública, Correos Electrónico: jovalle@sanmateo.edu.co
} 


\section{Resumen}

La presente investigación pretende establecer la situación de la organización World Visión en el ámbito contable, permitiendo diagnosticar el manejo de los inventarios, a través de un estudio. El fin es implementar un sistema de inventario permanente a través del método de promedio ponderado para brindar una mejor administración en las donaciones que son entregadas en eventos a las personas en condición de vulnerabilidad. La investigación en este proyecto es de tipo descriptivo, requiere de un diseño cuantitativo llevado a un estudio de caso, en el cual se realiza una macro con el programa Microsoft Excel, para ser implementado al programa contable SunSystem en la organización World Visión. De esta manera, se podrá conocer el costo de las donaciones y la cantidad que se encuentra en existencia, mejorando en la gestión contable más específicamente en el área de inventarios.

Palabras clave: world visión; inventario; método promedio ponderado; macros; contabilidad.

\section{Abstract}

The following project aims to establish the situation of the World Vision organization in the accounting field, allowing to diagnose inventory management, through a study. The purpose is to implement a permanent inventory system by the weighted average method, to provide a better administration in donations that are delivered in events to people in a condition of vulnerability. The research in this project is descriptive with a quantitative design, since the study is based on a specific sample, taken to a case study, in which a macro is made with the Microsoft Excel program, to be implemented to the Sun System accounting program in the World Vision organization. In this way, you can know the cost of donations and the amount that is in existence, improving in the accounting management more specifically in the area of inventories.

Keywords: world vision; inventory; weighted average method; macros; accounting.

\section{Introducción}

En la actualidad uno de los aspectos más importantes a controlar son los inventarios que representan varias operaciones y por lo tanto tienen un papel fundamental en la organización (Suárez-Pineda, Franco, Acosta-Triviño, y Alonso, 2017). Desde este punto de vista, la organización World Visión es una organización cristiana humanitaria de desarrollo, 
dedicada a trabajar con la niñez, juventud y comunidades para reducir la pobreza. Debido a esto, su principal problema es la inexistencia de control en la entrada y salida de los inventarios que regule el control interno y externo en la distribución de los kits escolares o las donaciones físicas que realizan en productos varios como: lápices, cuadernos, juguetes, refrigerios, destinados a las personas de bajos recursos económicos. Las entradas se dan mediante las donaciones en efectivo o en especie por otras organizaciones, empresas o personas, ya que se realizan registros contables en partida doble sin reconocer las existencias físicas o las salidas en conteo.

La presente investigación se enfoca en crear una macro o un apoyo para la implementación del sistema de inventario permanente. El manejo de inventarios en una organización, entidad o empresa permite el suficiente control sobre los materiales y recursos con los que se cuenta y que pueden ser usados en cuanto se requieran. En el caso de la organización World Visión es de vital importancia conocer las cantidades exactas de las donaciones como lo son kits escolares, juguetería, refrigerios en existencia, con el objetivo de evitar la pérdida de las donaciones o kits que se entregan en los eventos que realiza la empresa.

Granados (s.f.) corrobora que los sistemas de inventario permanente "son inventarios físicos y su función es verificar la existencia de los activos y/o mercancías de la empresa, sean inventario general o el inventario parcial como una clase de inventario que se hace sobre determinados rubros" (p. 61).

Esta organización no cuenta con una herramienta para llevar a cabo la administración de los inventarios que permita controlar y suministrar la cantidad de los elementos pertenecientes a la organización. Lo anterior dificulta conocer los artículos como lo son las donaciones que brindan las entidades o empresas, de modo que se pretende evitar la pérdida de estos elementos o productos.

Con base en lo anterior, una macro es un conjunto de comandos que guarda Excel y se encuentran disponibles en el momento en que se va a ejecutar el inventario. De esta manera, permite obtener el resultado de los productos que hay en existencia y, de igual forma, conocer el costo de la mercancía, favoreciendo los ámbitos de la administración y el área contable, así como las personas que buscan crear un negocio y necesitan llevar un registro de su mercancía.

Por otro lado, minimiza el tiempo al ser utilizado en otras funciones para llevar a cabo un trabajo eficaz y de gran rendimiento tanto para los trabajadores como para la organización o entidad y, así, dar cumplimiento a la planeación en los procedimientos que se van a desarrollar, en el servicio que va a prestar la organización World Visión a sus clientes. 
Es importante recordar, lo importante y necesario de contar con un método de control de inventario, que le ayude a la empresa a mantener un registro diario, de la existencia en sus productos, que le permitan tener el control estimado sin caer en contratiempos (Serna, 2017).

Esta investigación es de gran importancia debido a que se va a manejar Microsoft Excel 2016, un programa diseñado para la creación, modificación y creación de macros para el sistema de inventario permanente a implementar, como soporte en el programa contable SunSystem. Este es utilizado por la entidad para interpretar, almacenar, controlar y administrar los inventarios de manera confiable y sistematizar el manejo y control de las entradas y salidas de las donaciones o kits que deben ser suministrados por la organización World Visión.

De acuerdo a lo anterior, es importante llevar a cabo los inventarios para llevar el control y el registro de la mercancía o productos que tiene una empresa, entidad u organización como World Visión. Las entradas, que son los kits que donan las empresas, deben ser suministrados para que al momento de la salida. Es decir, cuando se entregan a los niños y niñas en los eventos realizados, tener presente la cantidad que ingresa y sale para así satisfacer la necesidad de cada uno de las personas que quieren obtener este beneficio. Forero (2015) aclara:

Existen costos que no pueden ser incluidos como un mayor valor del inventario, tales como: cantidades anormales de materiales de desperdicio; horas ociosas u otros costos de producción, costos indirectos, que no contribuyan a traer al inventario a su locación y condición actual. (p. 83)

Una macro es un programa con un grupo de herramientas e instrucciones que pueden ser configuradas en aplicaciones como Microsoft Word o Excel. Para realizar la macro a implementar en la organización World Visión se utiliza Microsoft Office Excel 2016, cuyo fin es mecanizar el inventario manejado de forma repetitiva, ya que World Visión recibe donaciones por parte de empresas públicas o privadas y así mismo lleva acabo eventos donde estos productos salen del inventario.

Dentro de las Organizaciones no Gubernamentales (ONG) podemos encontrar asociaciones religiosas que movilizan fondos privados para el desarrollo y la planificación familiar para fomentar la organización comunitaria, estas entidades que se encuentran en países industriales que apoyan el desarrollo internacional e incluso se encuentran grupos locales organizados en los planos nacionales. Según Oviedo (2017):

“La ONG analizada, con base religiosa, presenta flexibilidad y formas de control menos rígidas en la práctica, promoviendo mayor disposición por procesos de aprendizaje colectivos, aceptando ideales de otros y construyendo formas mejores para realizar sus actividades" (p. 542). 
Las macro se basan en un código de programación que permite digitar secuencias de órdenes realizadas de forma constante, como el inventario de los kits escolares, juguetería de la organización World Visión. Esta secuencia de ordenes se realizan por medio de un programa que tiene incorporado Excel Ilamado Visual Basic For Applications (VBA), un editor donde se programan las macros. De esta forma una macro permite automatizar o mecanizar el trabajo en Excel de forma manual, permitiendo establecer funciones y soluciones en el inventario permanente por el método de valuación promedio ponderado.

\section{Metodología}

El presente artículo se encuentra orientado hacia un diseño cuantitativo de tipo descriptivo, de manera que se basa en una muestra específica, llevado a un estudio de caso. Para implementar un sistema de inventario permanente, se realiza una macro en Excel que permita conocer el costo de las donaciones. Se evidencia que la empresa en cuestión no vende un producto, por el contrario, recibe donaciones y/o productos para ser distribuidos en la población, de acuerdo con lo establecido en su objeto social. Por lo anterior, se propone una metodología para poder establecer un control de inventario que a su vez sirva de soporte informático suministrando el costo.

Técnicas de recolección de datos

- Inventario actual de la organización World Visión 2019.

- Análisis de la macro realizada del sistema de inventario permanente para obtener la información de las donaciones.

\section{Resultados}

En el desarrollo de la investigación se logra obtener la macro realizada en Excel. Se evidencia que por medio de la macro pueden registrarse las donaciones que ingresan y salen de la organización, obteniendo como resultado final el costo de la transacción de las donaciones recibidas por empresas públicas o privadas. De esta manera, las organizaciones llevan un control de los inventarios de forma permanente y pueden obtener los resultados en el momento que lo deseen, mejorando en el área de inventarios y, de igual manera, en el ámbito contable. Así, a continuación, se describe el procedimiento realizado frente a la respectiva implementación del sistema permanente en la compañía. 
Inventario de la organización World Visión

Tabla 1. Inventario World Visión

\section{Referencia}

1124 kit juguete 1 para niños

1125 kit juguete 2 para niñas

1126 kit juguete 3 para bebes

A1124 kit de hogar aseo

A1125 kit de hogar electrodomésticos

A1126 kit de hogar vestuario

B1124 kit escolar: cuadernnos, esferos

B1125 kit escolar: reglas, colores

\section{Costo Unitario Cantidad}

$\begin{array}{ll}10.000 & 190 \\ 10.000 & 180 \\ 12.000 & 170 \\ 15.000 & 60 \\ 15.000 & 20 \\ 12.000 & 20 \\ 8.000 & 15 \\ 6.000 & 12\end{array}$

88.000
667

Fuente: World Visión, 2019a

El inventario realizado en la organización World Visión cuenta con ocho (8) referencias recibidas de las empresas públicas o privadas, las cuales suman 667 unidades con un valor de 88.000 pesos colombianos (Hayder A. C., 2019). En este sentido, se explicarán los sistemas de clasificación para organizar el inventario de la empresa World Visión.

Clasificación de inventarios en la organización World Visión

a) Clasificación por precio unitario

En este se clasifican los productos de acuerdo con el precio unitario de cada uno de los productos que hacen parte del inventario. En este se ordenan los datos de mayor a menor, de acuerdo con la columna de precio unitario, como se ve evidencia en la Tabla 2.

Tabla 2. Por Precio unitario

\section{Referencia}

A1124 kit de hogar aseo

A1125 kit de hogar electrodomésticos

1126 kit juguete 3 para bebes

A1126 kit de hogar vestuario

1124 kit juguete 1 para niños

1125 kit juguete 2 para niñas

B1124 kit escolar: cuadernnos, esferos

B1125 kit escolar: reglas, colores
Precio Unitario Cantidad Valor

$\begin{array}{ccc}15.000 & 60 & 900.000 \\ 15.000 & 20 & 300.000 \\ 12.000 & 170 & 2.040 .000 \\ 12.000 & 20 & 240.000 \\ 10.000 & 190 & 1.900 .000 \\ 10.000 & 180 & 1.800 .000 \\ 8.000 & 15 & 120.000 \\ 6.000 & 12 & 72.000\end{array}$

Fuente: World Visión, 2019b 
b) Clasificación por valor total

Se realiza una multiplicación de la cantidad de los artículos en existencia por el precio unitario de cada uno de los artículos. Los datos se organizan de mayor a menor, de acuerdo con el resultado obtenido, como se puede evidenciar en la tabla 3.

Tabla 3. Por valor unitario

\section{Referencia}

1126 kit juguete 3 para bebes

1124 kit juguete 1 para niños 1125 kit juguete 2 para niñas

A1124 kit de hogar aseo

A1125 kit de hogar electrodomésticos

A1126 kit de hogar vestuario

B1124 kit escolar: cuadernnos, esferos

B1125 kit escolar: reglas, colores
Precio Unitario Cantidad Valor

$\begin{array}{ccc}12.000 & 170 & 2.040 .000 \\ 10.000 & 190 & 1.900 .000 \\ 10.000 & 180 & 1.800 .000 \\ 15.000 & 60 & 900.000 \\ 15.000 & 20 & 300.000 \\ 12.000 & 20 & 240.000 \\ 8.000 & 15 & 120.000 \\ 6.000 & 12 & 72.000\end{array}$

Fuente: World Visión, 2019c

c) Sistema de inventario Permanente

El sistema de inventario permanente permite llevar el control de la mercancía existente en el tiempo que la organización lo requiera, mediante un control constante acerca de las donaciones que son recibidas por las demás entidades. De igual manera, permite determinar el costo al momento de realizar una donación pues en cada salida de una donación, se registra la cantidad y el costo.

Este control de la mercancía se realiza mediante una tarjeta llamada Kardex. En esta se lleva el registro de las referencias de los productos, la cantidad, su valor de compra, la fecha en la cual se adquirió la donación. Asimismo permite determinar la fecha en la cual sale el producto del inventario.

En el sistema de inventario permanente no es necesario llevar un registro del conteo físico de los productos. Las tarjetas Kardex permiten conocer las donaciones que han ingresado y asimismo cuántas han salido de la organización en cada evento realizado. Con estos datos se genera el valor actual del inventario en el momento que la organización lo requiera.

Una vez conocido el sistema de inventario permanente se pueden determinar dos (2) ventajas: 
- Se podrá conocer el saldo actualizado del inventario en cualquier momento que la empresa lo requiera.

- Permite obtener el costo de la mercancía en el momento en el que se produce la salida de las donaciones.

d) Método de evaluación de inventario

Los métodos de valuación son un conjunto de procedimientos utilizados con el fin de controlary estimar el flujo y el costo de la mercancía que ingresa y sale del inventario. Estos métodos son una herramienta que brinda información importante para tomar decisiones en el área administrativa y contable dentro de la organización World Visión. Para el presente artículo se maneja el promedio ponderado como método de valuación.

e) Método de evaluación por promedio ponderado

Este método se basa en valorar el promedio de la mercancía que compra o ingresa a la organización y aquella que se mantiene en existencia. Lo anterior se divide por el número de los productos, artículos, donaciones o kits y este valor será el asignado como costo unitario de las salidas de dichos artículos. Según Guzmán et al. (2005):

La valoración del inventario no depende del conteo físico de las unidades, el sistema de información debe ser tan eficiente y tan detallado que permita, a pesar de la gran cantidad de referencias o líneas de producto que puede llegar a manejar una empresa, saber exactamente cuantas unidades se tienen disponibles de cada una de las referencias o líneas de producto (p. 93).

Este método resulta útil para la organización, mantiene un control e identifica de manera precisa cuando se trata de los mismos artículos. De igual forma como el ingreso y la salida son aleatorios, con respecto a la adquisición, lo más ajustado es utilizar este método de valuación. Una vez aclarada la información acerca del sistema de inventario permanente por el método de valuación promedio ponderado, procedemos en cómo se realiza la macro para la ejecución de la prueba realizada.

\section{Conclusiones}

Dentro del análisis expuesto, es posible que World Visión tenga la información necesaria para manejar y tener clara la implementación del sistema de inventario. Con esta aplicación se facilita el proceso de llevar acabo el inventario de las donaciones. De igual forma, con el uso y el 
manejo de la macro que se realizó en excel, se logró obtener información verídica y de manera eficaz. Así, lo anterior tuvo una gran reacción en el poco tiempo en el que se lograba obtener la información de los kits que se encontraban en el almacenamiento y aquellos kits que llegaban para ser entregados en los eventos.

La búsqueda de información llevó a World Visión a tener iniciativa para llevar a cabo un inventario permanente que facilite el proceso al momento de tomar decisiones en los eventos que realizan para la entrega de las donaciones. Una vez conocidos los productos que hay en existencia, pueden conocer si pueden realizar los eventos en el momento que lo consideren necesario.

Con la implementación del sistema de inventario permanente por el método de valuación promedio ponderado, se realizó una prueba en la cual se comprobó que existe una mayor administración y control en los productos donados por otras empresas o entidades, bien sean publicas o privadas, en el cual la ONG World Visión se vio favorecida por este manejo de los kits escolares de juguetería, entre otros.

La prueba que se realizó en el proyecto para el manejo de la aplicación permite evidenciar que la macro es fácil de usar y entender, lo que hace que cualquier empleado de la organización World Visión pueda participar en su manejo, para ayudar a realizar las actividades sin ningún problema.

Por otro lado, la aplicación permite conocer las existencias de los kits y dar un informe en el cual se logra evidenciar que dichos kits a entregar, de manera adecuada, a aquellos niños vulnerables, sin tener perdidas. Además, existe un mayor control y administración, en el cual se logra satisfacer la necesidad tanto de los niños que esperan sus donaciones en los eventos realizados, como para la organización que logra obtener una satisfacción al ver a estos niños disfrutando de aquellas donaciones.

\section{Lista de referencias}

Forero, N. d. (2015). Implementación de norma internacional de inventarios en Colombia. Bogotá: INNOVAR.

Gorostegui, E. P. (2017). Curso de Economía de la empresa Introducción. España: Universitaria Ramón Areces.

Granados, I. (s.f.). Contabilidad Gerencial Fundamentos, principios e introducción a la contabilidad. Universidad Nacional de Colombia. 
Guzmán et al., A. D. (2005). Contabilidad Financiera. Bogotá: Centro Editorial Universidad del Rosario.

Hayder, A. C. (2019). Informe Inventario World Visión 2019. Bogotá: World Visión International.

Jaime, J. J. (2009). Contabilidad Financiera. Publicaciones de la Universitat Jaume.

Oviedo, A. B. (2017). La normatividad religiosa en una ONG: Experiencias negociadas de edad y género. Revista Latinoamericana de Ciencias Sociales, Niñez y Juventud, pp. 533-545.

Serna, José Mauricio, G. L. (s.f. de s.f. de 2017). Sistema de control de inventario. Obtenido de http://dspace.tdea.edu.co/bitstream/ tda/375/1/SISTEMA\%20DE\%20CONTROL\%20DE\%2 OINVENTARIO. pdf

Soto, C. A. (2011). Presupuestos empresariales. Bogotá: Proquest Ebook Central.

Suárez-Pinera, J., Franco, R., Acosta-Triviño, R. y Alonso, H. (2017). Nariño el contador, el precursor-libertador: la ilustración, la independencia. Bogotá: Escuela Militar de Cadetes "General José María Córdova"Universidad de San Buenaventur - Fundación Universitaria San Mateo. Recuperado de https://palma.sanmateo.edu.co/

World Vision International (2019a). Inventario de la Organización World Visión. World Vision. Recuperado de: https://www.worldvision.co/

World Vision International (2019b). Clasificación Por precio Unitario. World Vision. Recuperado de: https://www.worldvision.co/

World Vision International (2019c). Clasificación Por precio total. World Vision. Recuperado de: https://www.worldvision.co/ 